\title{
Recurrent and non-recurrent galactic cosmic-ray flux short-term variations observed with LISA Pathfinder
}

\author{
Simone Benella* \\ University of Urbino "Carlo Bo", Urbino, Italy and INFN, Sect. in Florence, Italy \\ E-mail: s.benella@campus.uniurb.it
}

\section{Catia Grimani}

University of Urbino "Carlo Bo", Urbino, Italy and INFN, Sect. in Florence, Italy

E-mail: catia.grimani@uniurb.it

\section{Michele Fabi}

University of Urbino "Carlo Bo", Urbino, Italy

E-mail: michele.fabi@uniurb.it

\author{
Noemi Finetti \\ University of L'Aquila, L'Aquila, Italy and INFN, Sect. in Florence, Italy \\ E-mail: noemi.finetti@cc.univaq.it \\ Mattia Villani \\ University of Urbino "Carlo Bo", Urbino, Italy and INFN, Sect. in Florence, Italy \\ E-mail: mattia.villani@uniurb.it
}

\begin{abstract}
Recurrent and non-recurrent galactic cosmic-ray (GCR) flux short-term variations, including three Forbush decreases, were observed with a particle detector aboard the European Space Agency mission LISA Pathfinder (LPF) orbiting around the Lagrangian point L1 between 2016 February 18 and 2017 July 3. The comparison of neutron monitor observations gathered at different geographic latitudes with contemporaneous LPF measurements provide precious clues about the energy-dependence of both recurrent and non-recurrent GCR flux short-term variations. A detailed study of the correlation between interplanetary structures and cosmic-ray short-term variations in 2016-2017 was also carried out and the results are presented here.
\end{abstract}

36th International Cosmic Ray Conference -ICRC2019-

July 24th - August 1st, 2019

Madison, WI, U.S.A.

${ }^{*}$ Speaker. 


\section{Introduction}

The galactic cosmic-ray (GCR) spatial distribution in the inner heliosphere is almost isotropic and the composition of these particles consists approximately of $90 \%$ protons, $8 \%$ helium nuclei, $1 \%$ heavy nuclei, and $1 \%$ electrons. GCR flux observations in the inner heliosphere present longterm (greater than 1 year) and short-term (less than 1 month) variations. For long-term variations, the overall GCR energy integral flux at $1 \mathrm{AU}$ ranges approximately from 4000 particles $\mathrm{m}^{-2} \mathrm{sr}^{-1}$ $\mathrm{s}^{-1}$ at solar minimum to 1000 particles $\mathrm{m}^{-2} \mathrm{sr}^{-1} \mathrm{~s}^{-1}$ at solar maximum, showing an 11 yr quasiperiodicity [1]. The flux modulation during cycles with opposite solar polarities shows a 22-yr quasi-periodicity (e.g., [2], and references therein). GCR short-term variations, are associated with the passage of recurrent and non-recurrent large-scale interplanetary structures $[3,4,5]$. The origin of recurrent variations lies in the interaction between GCR and corotating high-speed solar wind streams generated by coronal holes [6]. Quasi-periodicities of 27 days, 13.5 days, and 9 days, correlated with the Sun rotation period (27.28 days for an Earth observer) and higher harmonics, are indeed observed in the cosmic-ray flux, in the solar wind, in the interplanetary magnetic field (IMF), and in the geomagnetic activity indices [7]. The most intense short-term GCR flux nonrecurrent drops are called Forbush decreases (FDs) [8, 9] and are characterized by maximum GCR flux variations of $30 \%$ at $100 \mathrm{MeV} \mathrm{n}^{-1}$ associated with the passage of interplanetary coronal mass ejections (ICMEs).

This paper is organized as follows: in Sec. 2 the LISA Pathfinder (LPF) experiment is presented, in Sec. 3 the LPF data are shown: the recurrent and non-recurrent GCR flux short-term variations are studied and compared to solar wind, IMF and ground-based neutron monitor (NM) observations.

\section{The LISA Pathfinder experiment}

LPF was the technology demonstrator mission of the European Space Agency for LISA, the first space interferometer devoted to gravitational wave detection in space, exploring the frequency interval ranging between $10^{-4} \mathrm{~Hz}$ and $10^{-1} \mathrm{~Hz}$ [10]. The LPF spacecraft (S/C) was launched with a Vega rocket on 2015 December 3 from the Kourou base in French Guiana and reached its final orbit around the L1 Lagrangian point, at 1.5 million km from Earth in the Earth-Sun direction, at the end of January 2016. The elliptical orbit was inclined by about $45^{\circ}$ to the ecliptic and the S/C needed approximately six months to complete the orbit. Minor and major axes of the orbit were $\sim 0.5$ million $\mathrm{km}$ and $\sim 0.8$ million $\mathrm{km}$, respectively. The satellite rotated around its own axis in six months. The mission ended on July 2017. The LPF satellite payload carried two 2-kg cubic gold-platinum free-floating test masses (TMs) that played the role of mirrors of the interferometers for gravitational wave detection. The aim of the mission was to measure all the spurious forces affecting the TM acceleration recorded by the interferometers. In particular, it was of major importance to monitor the Coulomb forces induced by the TM charging due to the interactions of energetic particles of solar and galactic origin in the S/C material. Indeed, protons and ions with energies larger than $100 \mathrm{MeV} \mathrm{n}^{-1}$ interacted and/or penetrated the $13 \mathrm{~g} \mathrm{~cm}^{-2}$ of spacecraft and instrument materials charging the LPF TMs, [11]. In order to carry out a precise in situ monitoring of the overall flux of particles crossing the S/C, a particle detector (PD) was 
placed aboard LPF [12]. This PD was installed behind the spacecraft solar panels with its viewing axis oriented along the Sun-Earth direction. It consisted of two $\sim 300 \mu \mathrm{m}$ thick silicon wafers of $1.40 \times 1.05 \mathrm{~cm}^{2}$ area, placed in a telescopic arrangement at a distance of $2 \mathrm{~cm}$. For particle energies greater than $100 \mathrm{MeV} \mathrm{n}^{-1}$, the instrument geometrical factor was found to be constant and equal to $9 \mathrm{~cm}^{2} \mathrm{sr}$ for the incidence isotropic flux of particles on each silicon layer. A shielding copper box of $6.4 \mathrm{~mm}$ thickness surrounded the silicon wafers. The shielding material stopped particles with energies smaller than $70 \mathrm{MeV} \mathrm{n}^{-1}$. The PD allowed for the counting of protons and helium nuclei crossing each silicon layer and for the measurement of ionization energy losses of particles in coincidence mode. The single counts were gathered with a sampling time of $15 \mathrm{~s}$ and ionization energy losses of events in coincidence mode were stored in the form of histograms over periods of $600 \mathrm{~s}$ and sent to the on-board computer. The maximum allowed detector counting rate was 6500 counts $\mathrm{s}^{-1}$ on both silicon wafers, corresponding to an event integrated proton fluence of $10^{8}$ protons $\mathrm{cm}^{-2}$ at energies $>100 \mathrm{MeV}$. In coincidence mode, 5000 energy deposits per second was the saturation limit. The occurrence of solar energetic particles (SEPs) with fluences larger than the saturation limit was estimated to be less than one per year for the period the LPF spacecraft remained in orbit around L1 [13], though no SEP events characterized by a proton differential flux above a few tens of $\mathrm{MeV} \mathrm{n}^{-1}$ overcoming that of GCRs were observed during the period of the LPF mission operations.

\section{GCR short-term variations aboard LPF}

Short-term variations of the GCR flux observed in the inner heliosphere are due to interplanetary processes. The typical time scales for these variations range from several hours to one month. Nominal quasi-periodicities of 27, 13.5 and 9 days related to the Sun rotation period and higher harmonics are observed in the GCR flux. The whole GCR data sample gathered with LPF from 2016 February 18 to 2017 July 3 at a frequency of $0.067 \mathrm{~Hz}$ is shown in Fig. 1. Fortyfive recurrent variations, three FDs and 23 non-recurrent short-term variations shorter than two days are observed. The LPF data were hourly averaged in order to set the statistical uncertainty on each data point to $1 \%$. The percentage variation of these measurements calculated with respect to their average value observed during each Bartels rotation (BR) was visually inspected over the LPF mission lifetime. This approach was adopted in order to limit the role of the solar modulation decrease during the years 2016-2017. It is recalled here that the BR number represents the number of 27 day periods of the Sun since 1832 February 8 . The GCR flux variations were compared to contemporaneous IMF and solar wind plasma parameters gathered by the ACE experiment [14] orbiting around L1.

Recurrent GCR variations were studied by using the empirical mode decomposition (EMD) technique. The EMD is based on the assumption that any time series can be represented by a superposition of monocomponent signals $C_{i}(t)$, called intrinsic mode functions, that are characterized by a well-defined mean frequency [15]. Once all the intrinsic mode functions are known and subtracted from the original data set, the remaining function is called residue of the decomposition, $\operatorname{res}(t)$. The residue is, by construction, a monotonic function or a function with only one maximum and one minimum and represents the trend of the data. In Table 1 are reported the mean oscillation periods present in the LPF GCR data: intrinsic mode functions 7-9 show that periodicities of about 9, 13.5, and 27 days, related to the Sun rotation and higher harmonics, are present. The application 


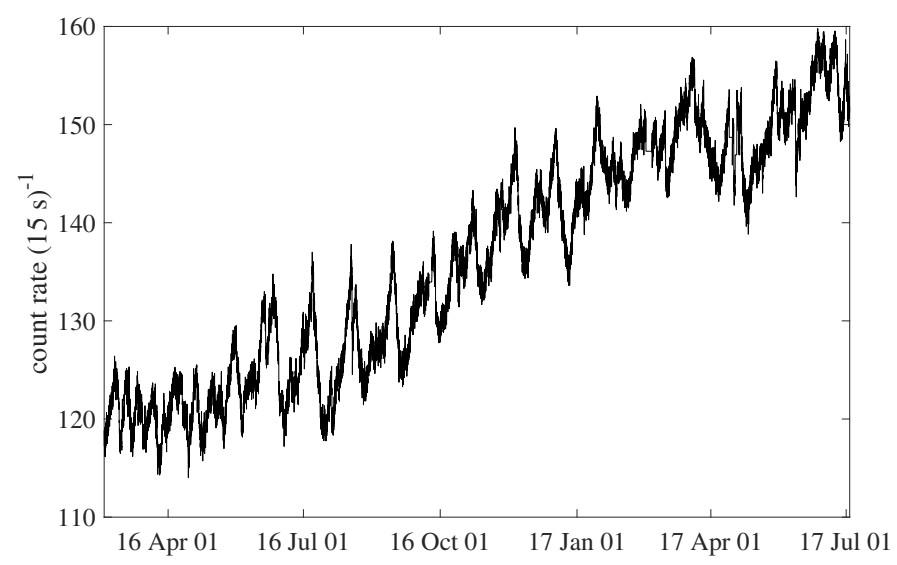

Figure 1: Hourly averaged GCR single count rate $(15 \mathrm{~s})^{-1}$ gathered by the LPF PD from 2016 February 18 to 2017 July 3.

of the $\mathrm{Wu}$ and Huang method [16] allows to quantify the statistical significance of intrinsic mode functions with respect to white noise. The intrinsic mode functions 6-11 lie above the 99\% white noise spread line. From the comparison between the residue of the EMD and the observed sunspot number is evident that the GCR count rate presents an increasing trend over the mission lifetime due to the decreasing solar activity, Fig. 2.

The average duration of the recurrent GCR flux short-term variations was found to be of $9.2 \pm 5.0$ days and the average intensity was of $5.1 \pm 2.5 \%$. The main role in generating short-term recurrent GCR flux variations was played by corotating interaction regions (CIRs) and high-speed solar wind streams (HSS) passage [5].

Three FDs were observed with LPF on 2016 July 20, Fig. 3, 2016 August 2, Fig. 4, and 2017 May 27, Fig. 5. The passage of three near-Earth ICMEs was associated with these FDs, as shown in the left panels of Figures 3-5. In these figures, GCR flux variations are compared to the solar wind speed, $V$, to the IMF sunward component with the opposite sign in the Geocentric Solar Ecliptic (GSE) coordinate system, $-B_{x}$, and to the IMF magnitude, $B$, gathered from the ACE

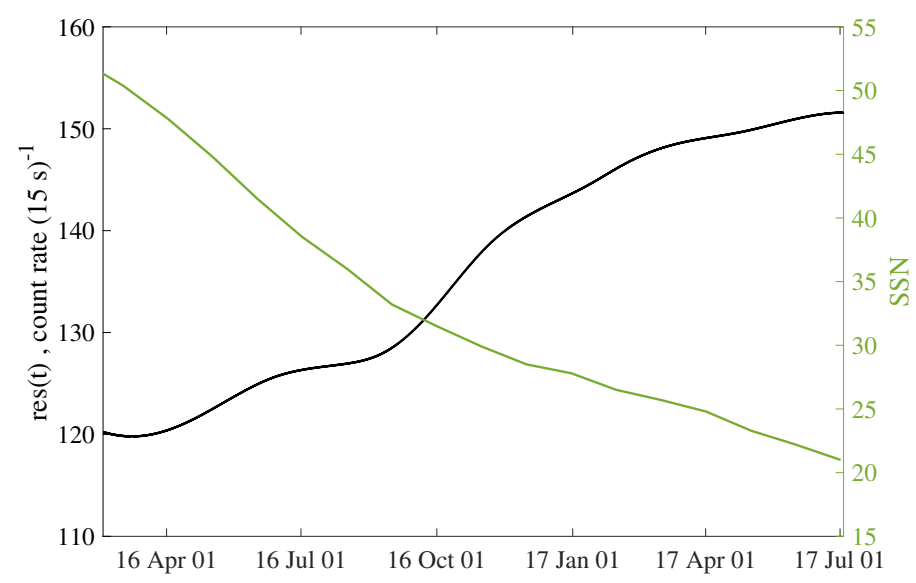

Figure 2: Comparison between the residue of the EMD and the 13-month smoothed monthly total sunspot number. The latter is taken as a proxy for the solar activity level. 
Table 1: Mean oscillation periods of the intrinsic mode functions from the EMD analysis on the GCR LPF data with standard errors.

\begin{tabular}{lclc}
\hline mode \# & mean period (days) & mode \# & mean period (days) \\
\hline 1 & $0.12 \pm 0.01$ & 7 & $10.3 \pm 0.3$ \\
2 & $0.21 \pm 0.01$ & 8 & $14.1 \pm 0.6$ \\
3 & $0.41 \pm 0.01$ & 9 & $27.6 \pm 1.0$ \\
4 & $0.81 \pm 0.01$ & 10 & $46.3 \pm 3.0$ \\
5 & $1.76 \pm 0.03$ & 11 & $87.9 \pm 7.1$ \\
6 & $3.8 \pm 0.1$ & & \\
\hline
\end{tabular}

experiment. The ICME passage is marked by dashed lines in the figures. The FD dated 2016 July 20 is associated with both solar wind speed and IMF increases due to the ICME propagating into a previous corotating high-speed solar wind stream (CHSS; $V>400 \mathrm{~km} \mathrm{~s}^{-1}$ ). On 2016 August 2
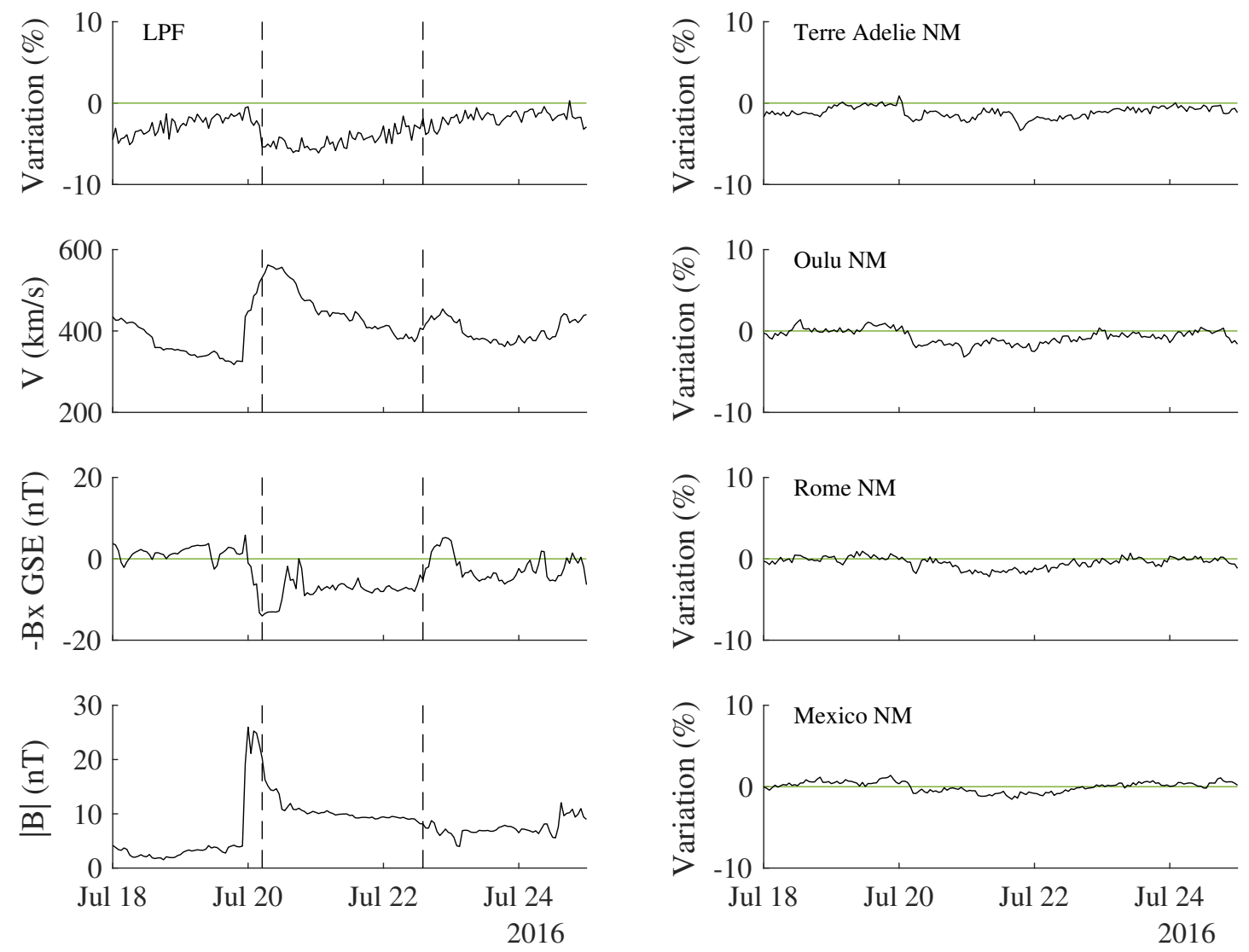

Figure 3: The LPF GCR flux percentage variations in the time interval 2016 July 18 - 25 are shown in the top panel of the left column and compared with $V,-B_{x}$ and $B$ ACE measurements. The vertical dashed lines indicate the ICME passage and the associated FD. NM measurements for the same time interval at different geographic latitudes (Terre Adelie: $66.65^{\circ} \mathrm{S}$, Oulu: $65.05^{\circ} \mathrm{N}$, Rome: $41.86^{\circ} \mathrm{N}$, Mexico: $19.33^{\circ} \mathrm{N}$ ) are shown in the right column. 

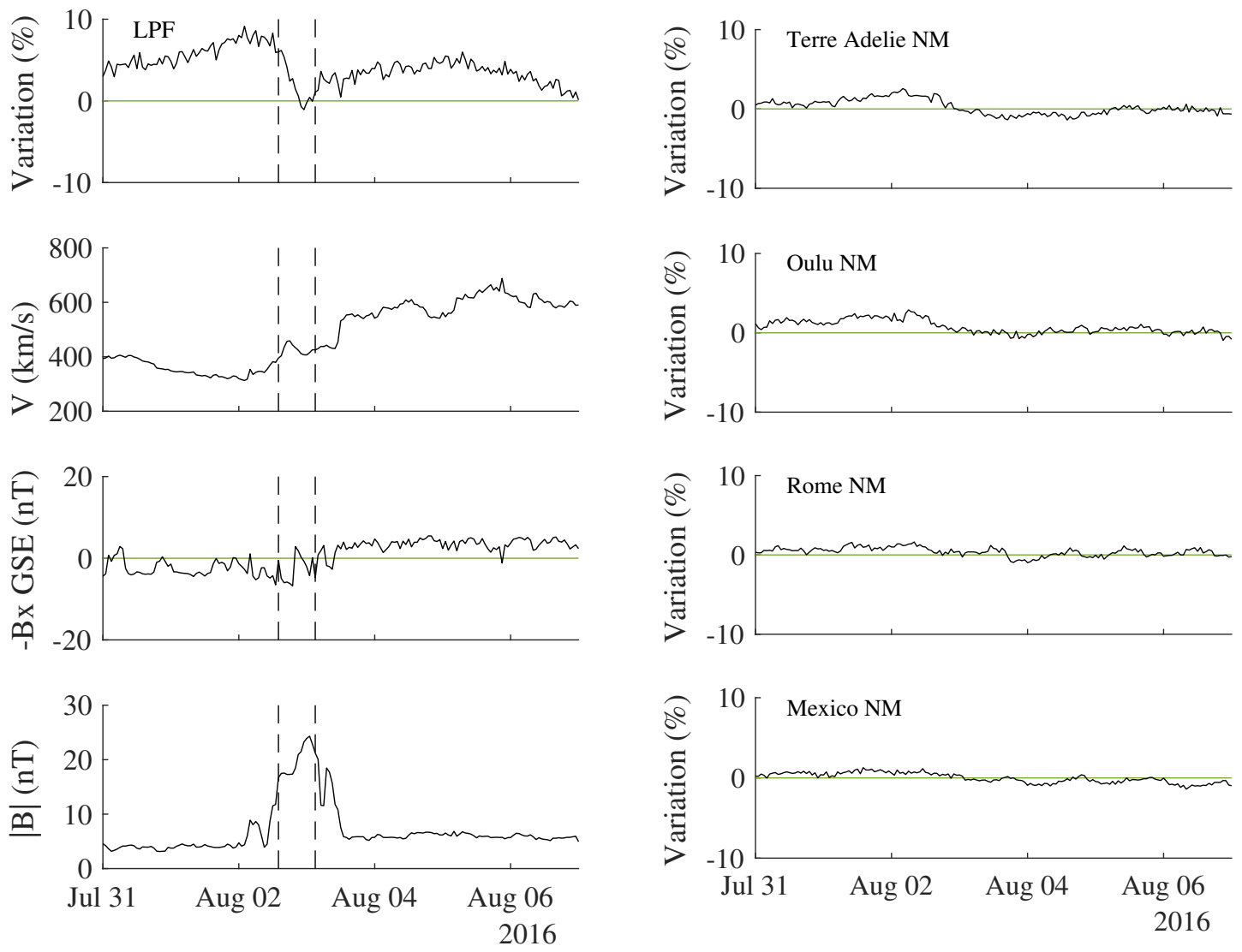

Figure 4: Same as Fig. 3 for the period 2016 July 31 - 2016 August 7.

and 2017 May 27 the GCR flux drops are associated to the IMF intensity increase due to incoming ICMEs. In all three cases the IMF intensity presented maximum values of about $25 \mathrm{nT}$. For the 2016 August 2 FD it was possible to investigate the role of the ICME magnetic ejecta in modulating the GCR flux [17]. In order to study the energy dependence of the three FDs observed with LPF, the percentage variation of the integral proton and helium fluxes measured with the PD above 70 $\mathrm{MeV} \mathrm{n}^{-1}$ was compared to hourly averaged variations of observations gathered with NMs located at different geographic latitudes (right panels of Figures 3-5). For a deeper discussion of this topic see [18].

From a visual inspection on the LPF PD data set, twenty-three non-recurrent short-term variations shorter than two days were observed between 2016 February 18 and 2017 July 3. GCR flux variations (depressions and peaks) considered in this analysis present a duration longer than 0.75 days (i.e., $18 \mathrm{hr}$ ) and intensities $>2 \%$ in order to set the statistical significance of the selection criterion to $2 \sigma$, given the $1 \%$ statistical uncertainty on PD hourly averaged single count data. The 23 observed variations consisted of 6 enhancements and 17 depressions. The majority of depressions $<2$ days in the GCR flux are associated with heliospheric current sheet crossings (HCSCs). CHSSs and/or CIRs appear scarcely correlated with this short GCR variations while recurrent short-term variations lasting more than 2 days do [5]. Peaks with duration $<2$ days appear to be associated with regions of compressed plasma between two CHSS. Several processes may generate these 

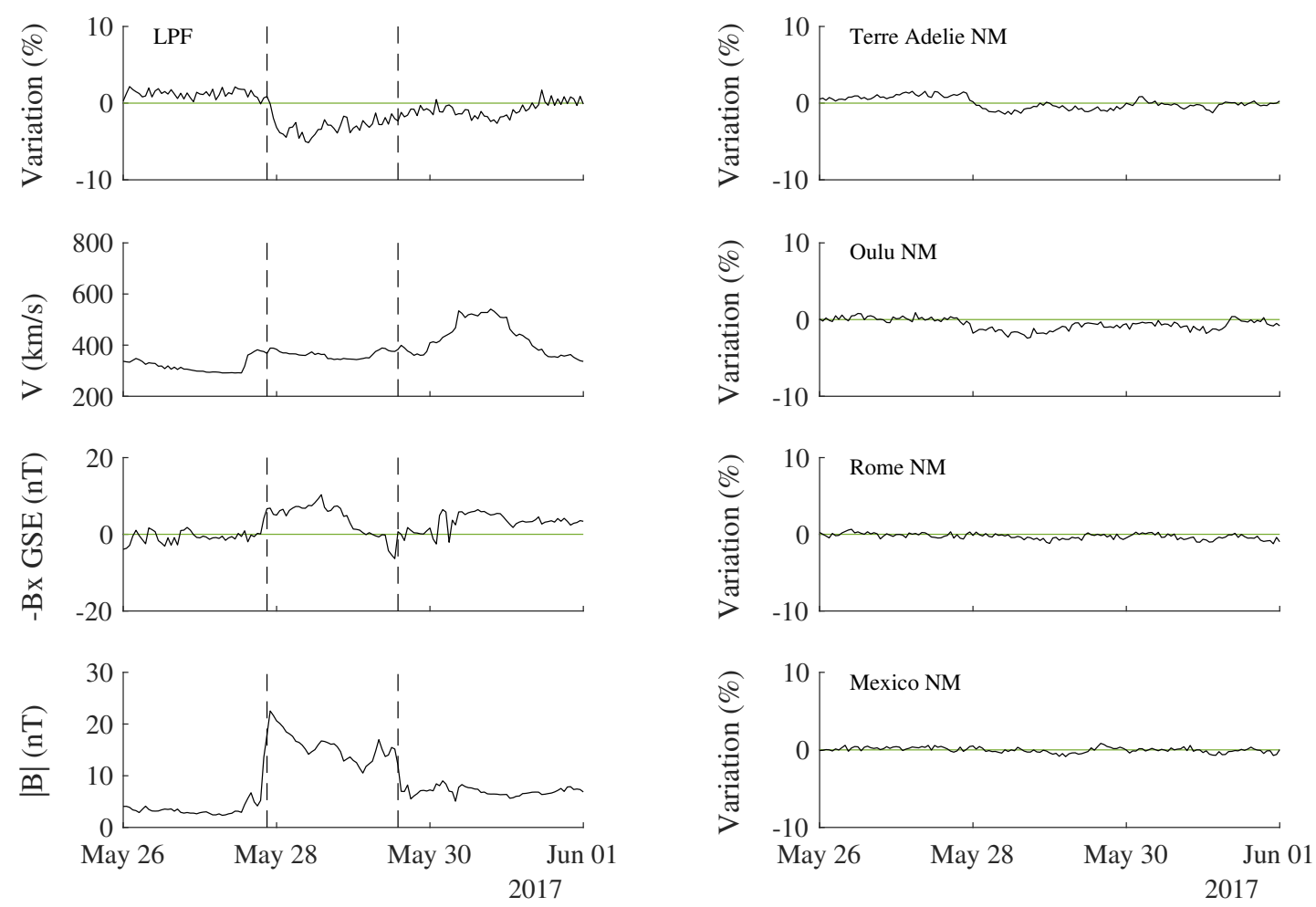

Figure 5: Same as Fig. 3 for the period 2017 May 26 - 2017 June 1.

small peaks in the GCR flux intensity. The most plausible is considered an effect of confinement of low-energy GCRs that cannot penetrate in regions of enhanced magnetic field intensity between subsequent CHSSs. However, a change in the low-energy GCR spectrum slope between a flux recovery phase after a CHSS passage and a second GCR flux decrease due to the passage of a subsequent CHSS, may also generate a peak feature in the observed integral flux. The acceleration process at the shock of incoming CHSS is not a valid candidate to explain the observed GCR peaks on the basis of the absence of small peak structures at the passage of isolated CHSS (see Figure 6 in [5]). Moreover, both models and observations indicate that the maximum energy of particles accelerated at CIR regions is about $20 \mathrm{MeV}$, far below the LPF low-energy cutoff [19, 20, 21].

\section{Conclusions}

A PD on board LPF allowed for the measurement of the integral flux variation of GCR protons and helium nuclei above $70 \mathrm{MeV} \mathrm{n}^{-1}$. The energy dependence of the FDs measured by LPF was compared to that of NMs, which allows for direct measurements of the integral flux variation of GCRs above effective energies $>10 \mathrm{GeV}$. Finally, hourly averaged GCR flux variations measured with LPF allowed for the observations of non-recurrent features in the GCR integral flux variations $>0.75$ days and $<2$ days with intensities $>2 \%$. These short-term depressions and peaks in the data trend appear to be correlated in the majority of cases with HCSC and plasma compression regions between subsequent CHSSs, respectively. 
The LPF data can be downloaded from https://www.cosmos.esa.int/web/lisa-pathfinder-archive/ home. Sunspot number data are gathered from http://www.sidc.be/silso/home. Data from the ACE and Wind experiments are taken from the NASA-CDAWeb website. The ICME catalog adopted in this work is available at http://www.srl.calctech.edu/ACE/ASC/DATA/level3/icmetable2.htm. NMs data are gathered from www.nmdb.eu and we thank the PIs of the NM network.

\section{References}

[1] Papini, P., Grimani, C., \& Stephens, A. S. 1996, NCimC, 19, 367

[2] Laurenza, M., Vecchio, A., Storini, M., \& Carbone, V. 2014, ApJ, 781, 71

[3] Richardson, I. G. 2004, SSRv, 111, 267

[4] Sabbah, I. 2000, GeoRL, 27, 1823

[5] Armano, M., Audley, H., Baird, J., et al. 2018a, ApJ, 854, 113

[6] Iucci, N., Parisi, M., Storini, M., \& Villoresi, G. 1979, NCim, 2C, 421

[7] Čalogović, J., Vršnak, B., Temmer, M., \& Veronig, Astrid, M. 2009, in Proc. IAU Symp. 257, Universal Heliophysical Processes, ed. N. Gopalswamy \& D. F. Webb (Cambridge: Cambridge Univ. Press), 425

[8] Forbush, S. E. 1937, PhRv, 51, 1108

[9] Cane, H. V. 2000, SSRv, 93, 55

[10] Amaro-Seoane, P., Audley, H., Babak, S., et al. 2017, arXiv:1702.00786

[11] Armano, M., Audley, H., Auger, G., et al. 2017, PhRvL, 118, 171101

[12] Cañizares, P., Chmeissani, M., Conchillo, A., et al. 2011, CQGra, 28, 094004

[13] Grimani, C., Boatella, C., Chmeissani, M., et al. 2012, CQGra, 29, 105001

[14] Stone, E. C., Frandsen, A. M., Mewaldt, R. A., et al. 1998, SSRv, 86, 1

[15] Huang, N.E. et al., Proceedings of the Royal Society of London A: mathematical, physical and engineering sciences, 454 (1998) 1971: 903-995.

[16] Wu, Z., Huang, N.E., Proceedings of the Royal Society of London A: mathematical, physical and engineering sciences, 460 (2004) 2046: 1597-1611.

[17] Benella, S. et al., 2019, NCimC, 42: 44

[18] Armano, M. et al., Astrophysical Journal, 874:167 (15pp), 2019 April 1

[19] McDonald, F. B., Teegarden, B. J., Trainor, J. H., von Rosenvinge, T. T., \& Webber, W. R. 1975, ApJL, 203, L149

[20] Tsurutani, B. T., Burton, M. E., Smith, E. J., \& Jones, D. E. 1985, Planet. Space Sci., 35, 289

[21] Giacalone, J., Jokipii, J. R., \& Kóta, J. 2002, ApJ, 573, 845 